Adecuación del Reglamento Específico del Sistema de Administración de Bienes y Servicios RE-SABS para el Gobierno Autónomo Municipal de Omerequ http://doi.org/10.33996/revistaenfoques.v1i3.14 No. 3 | Volumen 1 | Julio - Septiembre 2017 http://revistaenfoques.org ISSN: 2616 - 8219

\section{ADECUACIÓN DEL REGLAMENTO ESPECÍFICO DEL SISTEMA DE ADMINISTRACIÓN DE BIENES Y SERVICIOS RE-SABS PARA EL GOBIERNO AUTÓNOMO MUNICIPAL DE OMEREQUE}

\author{
ADEQUACY OF THE SPECIFIC REGULATIONS OF THE \\ RE-SABS GOODS AND SERVICES ADMINISTRATION SYSTEM \\ FOR THE AUTONOMOUS MUNICIPAL GOVERNMENT OF OMEREQUE
}

Norma María Zenteno Anturiano

\begin{abstract}
Resumen
La presente investigación fue realizada en el Gobierno Autónomo Municipal de Omereque (GAMO), éste presenta dificultades de tipo administrativofinanciero, debido a que los servidores públicos no tienen la experiencia y conocimiento suficiente en la aplicación de las disposiciones de administración y contratación de bienes y servicios, también manifiesta infracción a la normativa NB-SABS y la Ley 1.178. El objetivo de este estudio fue proponer la adecuación de un reglamento específico en el sistema de administración de bienes y servicios para el GAMO, con base en el modelo dispuesto por el órgano rector, un manual de procedimientos para los procesos de contratación y un programa de capacitación para su aplicación, con características y procedimientos específicos que se adecuen a la situación actual del municipio, que evite las responsabilidades estipuladas en la Ley 1.178 y sus decretos supremos reglamentarios. El tipo de estudio fue descriptivo y explicativo. La población de estudio fue de 35 servidores públicos del municipio y la muestra 4 de ellos del área de administrativafinanciera.
\end{abstract}

Palabras clave: Gobierno municipal; reglamentos específicos; administración de bienes y servicios

\begin{abstract}
The present investigation was carried out in the Municipal Autonomous Government of Omereque (GAMO), this presents difficulties of an administrative-financial type, because public servants do not have sufficient experience and knowledge in the application of the provisions of administration and contracting of goods and services, it also manifests a violation of the NB-SABS regulations and Law 1,178. The objective of this study was to propose the adaptation of a specific regulation in the system of administration of goods and services for the GAMO, based on the model provided by the governing body, a manual of procedures for contracting processes and a program of training for its application, with specific characteristics and procedures that adapt to the current situation of the municipality, which avoids the responsibilities stipulated in Law 1,178 and its supreme regulatory decrees. The type of study was descriptive and explanatory. The study population was 35 public servants of the municipality and the sample 4 of them from the administrative-financial area.
\end{abstract}

Key words: Municipal government; specific regulations; administration of goods and services
Norma María Zenteno Anturiano zentenorma@gmail.com Universidad Adventista de Bolivia, Bolivia

Lic. en Contaduría Pública de la Universidad Adventista de Bolivia. Técnico Superior en Contabilidad General, Diplomada en Educación Superior. Experiencia laboral en Administración Financiera y Planificación de presupuesto en gestión Municipal.

Artículo recibido abril 2017 | Arbitrado entre mayo-junio 2017 | Publicado en julio 2017 


\section{INTRODUCCIÓN}

Actualmente, las entidades públicas desarrollan sus actividades administrativas en el marco de la normativa gubernamental, con la finalidad de administrar los recursos del Estado con eficiencia, eficacia y sobre todo transparencia, las cuales son de cumplimiento obligatorio. Sin embargo, si bien estas son de cumplimiento obligatorio, se hace complicado cumplirlas al $100 \%$ por diferentes motivos, tal es el caso del Gobierno Autónomo Municipal de Omereque (GAMO), que se encuentra a una distancia considerable de los centros urbanos, además de la falta de normativa interna específica que se adecue a su propia realidad y la contratación de personal sin experiencia municipal que le está dificultando ejecutar el Plan Operativo Anual (POA) con eficiencia y eficacia.

Los trámites burocráticos y tediosos relativos a la contratación de bienes y servicios del GAMO están vulnerando la aplicación de las Normas Básicas del Sistema de Contratación de Bienes y Servicios y otras pertinentes, lo que genera responsabilidades a los servidores públicos tipificados en la Ley 1.178 (1990). Esto por la falta de capacitación y adiestramiento en la aplicación de la normativa a los servidores públicos de este municipio.

Por lo tanto, el objetivo de la investigación fue lograr que el municipio de Omereque pudiese contar con una normativa específica adecuada a su realidad y un programa de capacitación para el personal, a fin de lograr procesos de contratación, manejo y disposición de bienes ágiles y transparentes.

Los gobiernos autónomos municipales son entidades autónomas según establece el
Art. 283 de la Constitución Política del Estado Plurinacional de Bolivia, aprobado el 2010, y el Art. 34 de la Ley $n^{\circ} 031$ Marco de Autonomías y Descentralización Andrés Ibáñez, aprobado el 19 de julio de 2010 con derecho público, personalidad jurídica y patrimonio propio, cuyo propósito es la satisfacción de las necesidades de la comunidad. Sus recursos provienen principalmente de impuestos municipales, coparticipación tributaria, impuesto a los hidrocarburos, tasas, contribuciones especiales por mejoras, patentes y otros gravámenes señalados por ley.

El GAMO es la tercera sección de la provincia Campero del departamento de Cochabamba, creada bajo el gobierno de la presidenta Lidia Gueiler Tejada, mediante Ley $n^{\circ} 528$ de 9 de mayo de 1980, con sus cantones Huancani Grande, Peña Colorada, Pereta, Ele y Chaririri.

La administración de los gobiernos autónomos municipales, en busca de alcanzar el cumplimiento de los objetivos y metas trazadas, ejecutan los proyectos $y$ actividades planificados en el POA con recursos asignados por el Gobierno Central del Estado Plurinacional, y deben ser enmarcados en la normativa emanada a nivel nacional, departamental y municipal pertinente vigente.

El GAMO cuenta con una planta de profesionales según su organigrama para el desempeño de sus actividades programadas en la gestión. Sin embargo, la gran distancia que existe entre la ciudad de Cochabamba y el centro poblado de Omereque, así como el escaso transporte existente, hace que profesionales con poca o sin experiencia laboral accedan a trabajar en este municipio. Por otro lado, su falta o escaso conocimiento sobre la normativa del ámbito público, y más 
aún municipal, está ocasionando negligencias e incumplimiento a las disposiciones pertinentes legales, sobre todo en los procesos de contratación de bienes y servicios.

Por lo anteriormente expresado, se planteó el siguiente problema: ¿será que la aplicación correcta de la normativa específica en la administración de bienes y servicios en el GAMO logre un proceso efectivo y transparente en la administración y adquisición de bienes y servicios? Para contribuir a la administración eficiente y eficaz del GAMO mediante la adecuación de un RESABS (Reglamento Específico del Sistema de Administración de Bienes y Servicios) instrumento normativo propio de la entidad en cumplimiento a las normas vigentes - y que a través de un programa de capacitación a los servidores públicos del municipio se puedan mejorar sus conocimientos y obtener óptimos resultados en los procesos de contratación, manejo y disposición de los bienes y servicios del GAMO, se plantearon los siguientes objetivos específicos: adecuar procedimientos específicos de los tres subsistemas del RE-SABS que se ajusten a la realidad del GAMO; rediseñar el organigrama actual y ajustar al RE-SABS del GAMO; generar un manual de procedimientos con el apoyo de flujo gramas del subsistema de contratación de bienes y servicios con base en el RE-SABS; elaborar un programa de capacitación sobre el RE-SABS del GAMO para una mejor aplicación de la normativa.

\section{MATERIALES Y MÉTODOS}

El tipo de estudio realizado fue descriptivo-explicativo. Se definió, clasificó, catalogó y caracterizó el objeto de estudio. Con la finalidad de conseguir descripciones generales, se dirá que es de tipo lógico; y cuando la finalidad es la descripción de objetos específicos, diremos que es ideográfica.

Por otro lado, al saber que los estudios explicativos van más allá de la descripción de conceptos o fenómenos, o del establecimiento de relaciones entre conceptos, lo realizado estuvo dirigido a responder las causas de los eventos físicos o sociales, explicar por qué ocurren los hechos y en qué condiciones se dan estos, o por qué dos o más variables están relacionadas.

Los métodos de investigación utilizados fueron la observación, la deducción y el análisis. Las fuentes y técnicas utilizadas para la recolección de información fueron el cuestionario como fuente primaria, y como fuentes secundarias, textos y documentos.

\section{RESULTADOS Y DISCUSIÓN}

La siguiente tabla resume los resultados obtenidos. 
Tabla 1. Interrogantes y resultados de la investigación

\section{Interrogante}

¿El organigrama actual del municipio se adecua a su realidad administrativa?

¿Todos los servidores públicos están involucrados en la aplicación del SABS?

¿No se tiene el RE-SABS del GAMO homologado por el MEFP?

$¿ E$ El grado de conocimiento de las NB-SABS de los servidores públicos dificulta los trámites de contratación, control y disposición de bienes?

¿Procedimientos burocráticos en la aplicación del NB-SABS?

¿Los servidores públicos del GAMO no reciben capacitación sobre el NBSABS?

¿No se realizan inventarios de almacenes y activos fijos en GAMO?

¿Los servidores públicos desconocen los activos a su cargo?

¿Existe control de entradas y salidas de los insumos y activos fijos en almacenes?

\section{Resultados}

El $50 \%$ de las respuestas indica que el organigrama está de acuerdo en que se adecua a su realidad, y el $50 \%$ está en desacuerdo. Al no ser estas respuestas indicadores aceptables, se concluye que el organigrama no se adecua a su realidad.

El $75 \%$ de las respuestas indica que están totalmente de acuerdo con que todo el personal está involucrado en la aplicación del SABS y $25 \%$ muy de acuerdo. Las respuestas confirman que una gran mayoría de los servidores públicos están involucrados en el cumplimiento y aplicación de las NB-SABS.

El $100 \%$ de las respuestas indica que están totalmente de acuerdo con que no existe un RE-SABS homologado por el MEFP. Los servidores públicos entrevistados reconocen la inexistencia del RE-SABS del GAMO y más aún homologado por el MEFP. En conclusión, no existe un RE-SABS del GAMO homologado por el MEFP.

El $25 \%$ de las respuestas indica que están totalmente de acuerdo, el $50 \%$ muy de acuerdo y el $25 \%$ de acuerdo en que es el grado de conocimiento del SABS lo que dificulta la contratación, manejo y disposición de bienes. Las respuestas son variadas; sin embargo, se tiene una tendencia hacia que el grado de conocimiento del SABS del personal es el motivo que dificulta los trámites de contratación manejo y disposición de bienes.

El $75 \%$ de las respuestas indica que están de totalmente de acuerdo, mientras que el $25 \%$ está muy de acuerdo en que los procedimientos que se aplican en las NB-SABS son burocráticos. Las respuestas se inclinan a que los procedimientos aplicados en la NB-SABS son burocráticos; además, existen vacíos que lo dificultan aún más. En conclusión, los procedimientos que se aplican sobre las NB-SABS son burocráticos.

El $75 \%$ de las respuestas indica que están totalmente de acuerdo y el $25 \%$ muy de acuerdo en que los servidores públicos no reciben capacitación sobre las NB-SABS. Por la distancia a la que se encuentra este municipio es difícil la asistencia de los funcionarios a las capacitaciones en la ciudad, lo que torna difícil su participación y capacitación.

El $100 \%$ de las respuestas indica que están totalmente de acuerdo en que el personal de activos fijos y almacenes no efectúan inventarios periódicos. Dada la contundencia de las respuestas, se concluye que no se Ileva a cabo este protocolo, hecho que contraviene las NB-SABS.

El $75 \%$ de las respuestas indica que están totalmente de acuerdo y el $25 \%$ muy de acuerdo en que los servidores públicos no conocen los activos a su cargo y la responsabilidad que conlleva, hecho que contraviene las NB-SABS. En conclusión, los servidores públicos del GAMO conocen parcialmente su responsabilidad por tener un activo a su cargo. El $75 \%$ de las respuestas indica que están de totalmente de acuerdo y el $25 \%$ muy de acuerdo en que existe control de salidas e ingresos de almacenes y activos fijos. Las respuestas aseveran que existe control en salidas e ingresos de almacén y activos fijos en el municipio, pero no existe inventarios actualizados. 
Resumiendo los resultados obtenidos de los estudios realizados al GAMO referente a la aplicación del SABS, se puede indicar que existe una serie de factores que desfavorecen y muy pocas que favorecen a la aplicación de la normativa indicada: falta de conocimiento de la normativa por parte de los servidores públicos; procedimientos burocráticos y tediosos; contratación de personal sin experiencia; falta de capacitación permanente; abandono de algunos servidores públicos el municipio por la gran distancia hasta la ciudad; falta de normativa específica relativo al SABS que se adecue a la realidad del municipio.

Estas falencias actualmente son situaciones que entorpecen una óptima administración y, por ende, incide en la baja ejecución presupuestaria del POA. Es por eso que se propone solucionar estas dificultades con el diseño del RE-SABS, un flujograma de procedimientos para el GAMO que se adecue a su realidad actual y un programa de capacitación sobre el RE-SABS que haga fácil la aplicación de los procedimientos a todo el personal inmerso en ella.

Haciendo una interpretación o análisis de los datos arriba descritos referente a las respuestas recibidas, podemos indicar en algunos casos una información no muy alentadora, ya que los datos internos demuestran muy bajas opciones sobre cómo podría el municipio optar o encarar el tema de la aplicación del SABS. La fortaleza es el de contar con la predisposición de capacitar al personal por parte de las autoridades municipales. Dentro de las oportunidades con la que se puede contar es que los técnicos de AMDECO, a petición de las autoridades municipales, puedan trasladarse al municipio a impartir capacitación por lo menos 2 veces al año. Además, deben contar con el servicio de internet, puesto que a través de este medio los servidores públicos tienen acceso a la información actualizada sobre asuntos referidos a la normativa requerida.

Las variables externas, debilidades y amenazas, son diversas al contrario de las anteriores, pero las que se pueden destacar son la gran distancia que existe entre el municipio de Omereque y la ciudad de Cochabamba, la contratación de personal sin experiencia, bajos sueldos y la falta de transporte regular interprovincial. Estos factores inciden en la elevación de costos de insumos y materiales, el constante abandono del personal por la distancia y los bajos sueldos, y los resultados de la baja ejecución presupuestaria del POA.

En cuanto a los resultados de las entrevistas a los cuatro servidores públicos inmersos en la aplicación del SABS, la mayoría de las respuestas indica que existen falencias en la aplicación de la normativa en cuestión, debido a factores como la falta de conocimiento de la normativa, los procedimientos burocráticos, la falta de capacitación, la contratación de personal sin experiencia, la falta de una normativa específica del municipio referente a la contratación y control de bienes y servicios y estrategias que coadyuven a agilizar $y$ comprender a cabalidad la normativa y su aplicación menos burocrática.

\section{Discusión}

Existen diferentes definiciones sobre la administración pública. Una de ellas explica que es una ciencia social que tiene por objeto la actividad del órgano ejecutivo cuando se trata de la realización o prestación de los servicios públicos en beneficio de la comunidad, buscando siempre que su prestación se lleve a cabo de manera 
racional a efecto de que sus medios e instrumentos se apliquen de manera idónea para obtener el resultado más favorable por el esfuerzo conjunto llevado a cabo.

La administración pública es un sistema dinámico integrado por normas, objetivos, estructuras, órganos, funciones, métodos y procedimientos, elementos humanos $y$ recursos económicos y materiales a través del cual se ejecutan o instrumentan las políticas y decisiones de quienes representan o gobiernan una comunidad políticamente organizada (Carrillo, 2000).

La administración pública es un elemento básico del Estado. Está formado por un conjunto de organismos que actúan bajo órdenes del Poder Ejecutivo, encargados de dictar y aplicar las disposiciones necesarias para el cumplimiento de normativas, el fomento de los intereses públicos y la resolución de las reclamaciones de los ciudadanos que se produzcan (De la Encarnación, 2003).

La administración pública tiene por objeto utilizar eficaz y eficientemente los recursos de la sociedad puestos a su disposición, eliminar el despilfarro, reducir gastos, conservar y utilizar con criterio las máquinas y edificios, así como proteger el bienestar y el interés de los servidores públicos (Galindo, 2000).

El enfoque sistémico es una forma de pensar que permite ver el objeto de estudio, en este caso la administración pública como un sistema y su forma de administración también como un sistema.

En este mismo orden de ideas, un sistema es un conjunto de elementos relacionados entre sí y que funcionan como un todo. Los elementos que componen un sistema pueden ser variados, como una serie de principios o reglas estructurados sobre una materia o teoría. Según Amaro, es el "conjunto organizado, formando un todo en el que cada una de sus partes está integrada a través de una ordenación lógica, que encadena sus actos a un fin común" (Guzmán, 1993).

Desde el sistema de programación de operaciones hasta el sistema de control de contabilidad, el órgano rector es el Ministerio de Economía y Finanzas. Además de estos dos sistemas grandes, el órgano rector es el Ministerio de Planificación del Desarrollo, y del sistema de control el órgano rector es la Contraloría. Vale decir que tenemos tres órganos rectores.

Es necesario diferenciar claramente los conceptos: capacitación, entrenamiento y adiestramiento. El entrenamiento es la preparación que se sigue para desempeñar una función; el adiestramiento es el proceso mediante el cual se estimula al trabajador a incrementar sus conocimientos, destreza y habilidad y la capacitación es la adquisición de conocimientos técnicos, teóricos y prácticos que van a contribuir al desarrollo del individuo en el desempeño de una actividad.

La capacitación en la actualidad representa para las unidades productivas uno de los medios más efectivos para asegurar la formación permanente de sus recursos humanos respecto a las funciones laborales que deben desempeñar en el puesto de trabajo que ocupan. Si bien es cierto que la capacitación no es el único camino por medio del cual se garantiza el correcto cumplimiento de tareas y actividades, sí se manifiesta como un instrumento que enseña, desarrolla sistemáticamente y coloca en circunstancias de competencia a cualquier persona.

Asimismo, el manual de procedimientos es un componente del sistema de control interno, el cual se crea para obtener una 
información detallada, ordenada, sistemática

e integral que contiene todas las instrucciones, responsabilidades e información sobre políticas, funciones, sistemas y procedimientos de las distintas operaciones o actividades que se ejecutan en una organización.

Por su parte, el Proceso Administrativo del Sistema de Contratación de Bienes y Servicios es el proceso administrativo que logra el perfeccionamiento de los contratos, orden de compra y orden de servicio en las entidades públicas, con el objeto de adquirir u obtener un bien, la prestación de un bien, la prestación de un servicio y la ejecución de una obra, de acuerdo con el POA y el PAC, de tal manera que se obtenga bienes y servicios, con calidad, cantidad y precio justo, para lograr un beneficio equilibrado entre la entidad contratante $y$ el contratado proveedor (Sistema de Administración de Bienes y Servicios, Contraloría General de la República de Bolivia, 2009).

Igualmente, el Reglamento Específico del Sistema de Administración de Bienes y Servicios (RE-SABS) es un documento institucional en el que se particularizan conforme a las características y estructura de la entidad, los procesos, previsiones, funciones $\mathrm{y}$ responsabilidades establecidas en las Normas Básicas del SABS.

De acuerdo con lo dispuesto en la Ley 1.178, cada entidad para implementación del sistema deberá elaborar su propia reglamentación específica. Este documento deberá ser compatibilizado por el órgano rector, para evitar que se encuentre fuera de las NB-SABS. En el caso del SABS, el órgano rector establece la elaboración del reglamento específico, el cual, después de su compatibilización, la entidad pública deberá aprobar mediante resolución administrativa, según establece el Art. 9 del D.S. no 29190.
Ahora bien, las empresas en todo el proceso de diseñar e implementar el sistema de control interno, debe preparar los procedimientos integrales de procedimientos, los cuales son los que forman el pilar para poder desarrollar adecuadamente sus actividades, estableciendo responsabilidades a los encargados de las todas las áreas, generando información útil y necesaria, estableciendo medidas de seguridad, control y autocontrol, $y$ objetivos que participen en el cumplimiento con la función de la entidad. El sistema de control interno, aparte de ser una política de gerencia, se constituye como una herramienta de apoyo para las directivas de cualquier institución para modernizarse, cambiar y producir los mejores resultados, con calidad y eficiencia.

En razón de esta importancia que adquiere el sistema de control interno para cualquier entidad, se hace necesario hacer el levantamiento de procedimientos actuales: responsabilidad como la autoridad o delegación de funciones dentro del proceso; medidas de seguridad y autocontrol aplicables al procedimiento; informes económicos, financieros, estadísticos y recomendaciones; supervisión, evaluación y examen; entidades de control y gestión de autocontrol. Estos manuales se desarrollan para cada una de las actividades $u$ operaciones que tengan que ver con los procesos administrativos y operativos, de acuerdo con los lineamientos y exigencias establecidas por la ley, los cuales son el punto de partida y el principal soporte para llevar a cabo los cambios que con tanta urgencia se requieren para alcanzar y ratificar la eficiencia, efectividad, eficacia y economía en todos los procesos. 


\section{CONCLUSIONES}

Durante la ejecución de la investigación resumida en este artículo sobre el cumplimiento de la normativa que rige el área administrativa financiera GAMO, se debe entender que la gestión pública debe desarrollarse en un marco normativo institucional y administrativo de forma estricta en las entidades públicas, debido a la necesidad de garantizar principios que se consideran esenciales en la administración del Estado, como son la transparencia de las acciones públicas, la ecuanimidad, la disciplina fiscal y el uso apropiado de los recursos pertenecientes al patrimonio público.

Hay que considerar la burocracia existente en los sistemas de administración regulados en el marco normativo que caracteriza a la administración pública, porque indudablemente incide en los procedimientos y en la expresión de resultados concretos a nivel de la gestión. También influye la larga distancia existente entre el GAMO y los centros urbanos, los cuales abastecen de insumos y materiales a este municipio a través del comercio e industrias.

Además, a esto se le suma la contratación de servidores públicos sin experiencia por parte del municipio y el abandono de trabajo constante de algunos de ellos por el bajo costo de salarios. Y la falta de un reglamento específico del sistema de contratación y control de bienes y servicios que contemple los procedimientos específicos y adecuados al municipio y su respectiva capacitación. A pesar de estos factores negativos, el municipio debe ejecutar su presupuesto programado en la gestión y cumplir las disposiciones que rigen.

Por lo tanto, con la finalidad de dar una alternativa de solución a las dificultades que presenta este municipio referentes a los procedimientos de contratación y control de bienes y servicios, este proyecto de investigación desarrolló una adecuación del reglamento específico del SABS para el GAMO, basado en el modelo aprobado mediante Resolución Ministerial no 20 del 15 de enero de 2015 que aprueba los modelos de los reglamentos específicos del Sistema Gubernamental de la Ley 1.178 de los Gobiernos Autónomos Municipales. Dicha normativa interna proporciona a los servidores públicos procedimientos específicos en cada uno de sus subsistemas, tales como Subsistema de Contrataciones; Subsistema de Control de Bienes; Subsistema de Disposición de Bienes, instrumento normativo interno que será útil para normar los procedimientos más convenientes adecuados a su realidad, en el marco de las NB-SABS, con el objeto de mejorar, procurar y transparentar los trámites de contratación de bienes y servicios.

Asimismo, se propuso un rediseño del organigrama organizacional actual, que incluye al personal requerido para el desarrollo de los procedimientos propuestos, de tal forma que la unidad administrativa cuente con el personal requerido conforme estipula el reglamento propuesto.

$Y$ como instrumento normativo complementario al RE-SABS de Omereque, se plantea un manual de procedimientos $y$ sus flujogramas, lo cual constituye un instrumento normativo didáctico que comprende todos los pasos a seguir en cada una de las modalidades de contratación, los responsables que participan en cada trámite. Tiene la finalidad de proporcionar un documento de fácil comprensión para los servidores públicos del municipio que coopere con la agilidad y transparencia de los trámites en todas sus modalidades de contratación. 
Igualmente, un programa de capacitación y seguimiento para el personal del GAMO que logrará que los servidores públicos asimilen los procedimientos del RESABS en un tiempo breve y puedan aplicar y mejorar los procedimientos de las carpetas de contratación, manejo y disposición de los bienes del municipio. Además, los resultados de una mejor aplicación de la normativa en el GAMO en un plazo muy corto tendrán como consecuencia trámites dinámicos y transparentes por el cual la población del municipio estaría satisfecha y que en la siguiente gestión correspondiente a la elaboración del POA será de fácil consenso una planilla salarial con incremento salarial.

La aplicación de esta norma específica y su cumplimiento dependen de la cabeza de sector, es decir, de la MAE, del gobierno, el cual deberá asumir medidas de hecho en contra de los servidores públicos que incumplan estas disposiciones.

En estas líneas conclusivas, es preciso recomendar que para la aplicación del RESABS adecuado para el GAMO, primeramente la MAE deberá asumir acciones decididas, y coordinadas intrainstitucionalmente sobre las actividades a encaminar y los trámites a efectuar hasta obtener su correspondiente homologación ante el MEFP y su posterior difusión a cargo de los responsables definidos en el RE-SABS del GAMO.

La propuesta del rediseño del organigrama es un instrumento que les será útil aplicar en el POA, estructura que contempla tres cargos importantes para poder encarar los procedimientos del RESABS con mayor responsabilidad y holgura.

El manual de procedimientos adjunto a sus flujogramas facilitará la aplicación de los procedimientos en cada modalidad de contratación describiéndolos paso a paso con el apoyo de los flujogramas de forma didáctica. De esta manera, la MAE - previa utilización de la normativa indicada- deberá aprobarla mediante un Decreto Edil.

Finalmente, se recomienda a la MAE capacitar a los servidores públicos del GAMO, según la propuesta del programa de capacitación sobre el RE-SABS que servirá para mejorar y optimizar sus conocimientos y lograr un mejor rendimiento del personal, lo que elevará las capacidades y destrezas de los servidores públicos en la administración eficaz y eficiente de la cosa pública, basada en principios de transparencia e idoneidad profesional, así como la evaluación periódica de la asimilación de conocimiento del personal que aplica la normativa propuesta.

También es importante indicar lo siguiente: los ejecutivos y concejales del municipio deben incorporarse al proceso de aprendizaje del sistema de administración de bienes y servicios normados en el municipio, si desean fiscalizar y prestar servicios de calidad con transparencia y aceptación. Que como resultado de las capacitaciones al personal, lógicamente se mejorarían los resultados de la gestión municipal, por lo que debería considerarse el incremento salarial para poder especializar al personal en sus áreas, además de dar mejores condiciones de estadía del personal, ofreciéndoles viviendas y otras opciones que eviten la deserción de los servidores públicos.

\section{REFERENCIAS}

Carrillo, A. (2000). Teoría de la administración pública. México: Mc Graw Hill

De la Encarnación, A. (2003). Administración pública. España: Thompsom 
Galindo, M. (2006). Teoría de la administración pública. México: Editorial Porrúa

Guzmán, R. (1993). Introducción a la administración pública. México: Mc Graw Hill

Jiménez, W. (2007). Introducción al estudio de la teoría administrativa. México: Editorial Lirnusa, Noriega Editores
Ley 1.178 del 20 de julio de 1990, La Paz, Bolivia

Sistema de Administración de Bienes y Servicios (2009). La Paz: Contraloría General de la República

Sistema de Administración de Personal (2003). La Paz: D.S. 26115 